\title{
RTE-Extremadura.org: un portal de acceso al Sistema Educativo de Extremadura
}

\author{
Felipe Zapico Alonso \\ Universidad de Extremadura \\ José Antonio León Moreno \\ Dirección General de Sociedad de la Información \\ Junta de Extremadura
}

\subsection{Resumen}

Se presenta la génesis, desarrollo y mantenimiento del portal de la Educación (Rte-Extremadura.org) del Gobierno Autónomo de Extremadura (España), dirigido a la comunidad educativa y a los ciudadanos interesados. Sus objetivos específicos son proporcionar una visión global de las actividades e iniciativas y facilitar su seguimiento, ser un punto de encuentro e interacción entre los agentes educativos de la región (personal docente, padres y alumnos), investigar las acciones y políticas que en materia educativa se están desarrollando y los marcos legislativos en que se amparan, construir un espacio virtual de recursos didácticos de interés para la comunidad educativa, difundir y canalizar la información y comunicación sobre el uso de las tecnologías de las información y la comunicación (TIC) en Extremadura y el resto del mundo, fomentar actividades de formación en TIC para su posterior aplicación en el aula, y promover el uso de las TIC en la región mediante la colaboración de sus distintos sectores e instituciones. Se pretende crear una estructura básica que posibilite a sus usuarios insertar sus propios contenidos para hacerlo lo más participativo e interactivo posible mediante un formulario de alta de recursos. Para ello, se ha buscado un sistema de organización, inclusión y localización de información lo más dinámico, fácil y rápido posible, sustentado por bases de datos relacionadas. Para velar por la calidad de los contenidos, está prevista la creación de un comité de normalización y de evaluación.

Palabras clave: Red Tecnológica Educativa. Extremadura. Organización del Conocimiento. Portales educativos.

\subsection{Abstract}

The creation, development and maintainance of educational portal (RteExtremadura.org) of the Autonomous Government of Extremadura (Spain) is Scire. $9: 2$ (jul.-dic. 2003) 161-168. 
presented. The portal is addressed to the educational community and interested citizens, and its aims are to provide a global vision of the activities and initiatives and a meeting point among education professionals, parents and students; to study the actions and policies being developed and their legal background; to offer teaching resources of interest to the educational community; to disseminate and channel information and communication on information technologies (IT) between Extremadura and the rest of the world; to promote IT training and application in the classroom; and to promote the use of the IT in the region, through collaboration among its various sectors and institutions. A basic structure has been created so that users can include their own contents through a form. In order to achieve this a structure has been made available that enables people to activate any kind of resource or content, which they consider to be of interest to the community they addresses. The most dynamic, user-friendly and high-speed system for organizing, including and finding information has been sought, based on relational databases. A normalization and evaluation committee will be established to ensure the suitability and selection of the resources.

Keywords: Educational portals. Extremadura. Knowledge Organisation.

\section{Introducción}

El portal educativo de Extremadura - www.rte-extremadura.org - es el instrumento de información de la Red Tecnológica Educativa (RTE), una de las cuatro líneas estratégicas del Proyecto Global de la Sociedad de la Información para Extremadura. Su contexto amplio es el proyecto INFODEX, una estrategia de cooperación interregional creada en 1997 en el marco de la iniciativa Iniciativa Regional de Sociedad de la Información (RISI), y cofinanciada por la Unión Europea y la Junta de Extremadura a través de la Fundación para el Desarrollo de la Ciencia y la Tecnología en Extremadura (FUNDECYT). Sus objetivos eran aprovechar las aplicaciones de las tecnologías de la información para modernizar las distintas actividades productivas, mejorar los servicios de atención al ciudadano, disminuir las diferencias entre las zonas rurales y urbanas a través de las Tecnologías en Información y Comunicación (TIC) y actuar como región integradora de España y Portugal. En el marco de esta iniciativa, se diseñó el Proyecto Global de la Sociedad de la Información, cuyo Plan Estratégico consta de las siguientes líneas estratégicas:

1. Nuevos Centros del Conocimiento (NNC): Nace del compromiso del Gobierno Regional de desarrollar un Plan de Alfabetización Tecnológica (PAT) para toda Extremadura, que trata de crear un espacio de encuentro, físico y virtual, que convoque a distintos colectivos sociales para fomentar el desarrollo económico y sociocultural en la Comunidad Extremeña. 
Poro su parte, el PAT tiene como objetivo promover la sensibilización, el acceso, la alfabetización de los extremeños en las NTI.

2. Red Tecnológica Educativa de Extremadura (RTE): Es una red constituida por los agentes educativos, los recursos de información y las infraestructuras tecnológicas necesarias. Su objetivo es instalar alrededor de 90.000 puntos de red en toda la Región, para conseguir un ratio de un ordenador por cada 2 alumnos del sistema educativo extremeño. Esta acción posibilitará la incorporación del Sistema Educativo de Extremadura a la Sociedad de la Información.

3. Red Telemática Sanitaria (RTS): Pretende la conexión de todos los centros de salud y hospitales de la Región.

4. Viveros de Empresas para la Nueva Era (Vivernet): Es un espacio tecnológico en el que empresas o grupos de personas interesadas en llevar adelante proyectos en el ámbito de la Sociedad de la Información pueden desarrollar sus actividades empresariales.

Para facilitar el desarrollo de los programas mediante una estructura de comunicaciones avanzadas, la Junta de Extremadura convocó un concurso para contratar una Red Corporativa de Servicios Avanzados de Telecomunicación, el cual fue adjudicado a Retevisión en diciembre de 2000. En el año 2001 se comienza con el despliegue de la Intranet de Extremadura por parte de la empresa adjudicataria, con la elaboración de un anteproyecto técnico con los datos proporcionados por la Junta de Extremadura a través de sus Consejerías. De dicho anteproyecto, surge un plan de actuación y despliegue cuyos objetivos eran el desarrollo de la Intranet cubriendo una estimación inicial de 1.478 edificios con un acceso de datos requerido por edificio de $2 \mathrm{Mbps}$, proporcionando un acceso a Internet a un elevado número de usuarios concurrentes; y el desarrollo de un plan de numeración y despliegue de centralitas para el consumo de tráfico de voz en los edificios de la administración, incluyendo también a colegios e institutos. Con todo, ello se consigue unificar los servicios y tarifas de telecomunicaciones en toda la Junta, reduciendo los costes y mejorando los servicios de telecomunicaciones con las últimas tecnologías.

La Red de Servicios de Comunicaciones Avanzadas de la Junta de Extremadura o Intranet de Extremadura se constituirá como el soporte estratégico de numerosos procesos, culturales, educacionales y administrativos que contribuirán al progreso empresarial de la región y de los ciudadanos extremeños.

La implantación de servicios corporativos de comunicaciones y de aplicaciones en el conjunto de dependencias de la Administración Pública Autonómica, centros educativos y centros sanitarios de toda Extremadura implica la necesidad de disponer, en todos los centros, de accesos a redes de telecomunicaciones con

Scire. $9: 2$ (jul.-dic. 2003) 161-168. 
capacidad para satisfacer las necesidades que se generen en todos los estamentos dentro de la comunidad.

\section{RTE-Extremadura: objetivos y tecnología}

El motivo que ha llevado a concebir la creación de un portal de la Educación se debe a que es un espacio general que está englobado en la ya mencionada Red Tecnológica Educativa. Para el diseño de este portal se ha tenido en cuenta el usuario final al que va dirigido: los profesionales de la comunidad Educativa, incluyendo tanto a docentes como a discentes. El Portal de la Educación es un espacio de contenidos específicos en esta materia y un lugar de referencia informativa y participación de aquellos usuarios relacionados con las Tecnologías de la Información y la Comunicación (TIC). Sus objetivos son:

- Proporcionar una visión global y un espacio para el seguimiento de las actividades e iniciativas relacionadas con la Educación.

- Ser un punto de encuentro e interactividad entre los agentes educativos de la región, personal docente, alumnos y padres.

- Construir un espacio virtual de recursos didácticos de interés para la comunidad educativa.

- Difundir y canalizar la información y comunicación entre la comunidad educativa de Extremadura y el resto del mundo.

- Fomentar actividades de formación en TIC para su posterior aplicación en el aula.

- Promover el uso de las TIC en la región, mediante la colaboración de los distintos sectores e instituciones de la región.

Con el Portal se pretende crear la estructura básica que posibilite a sus usuarios insertar sus propios contenidos para hacerlo lo más participativo e interactivo posible por lo que se ha habilitado un formulario donde poder dar de alta cualquier tipo de recurso o contenido propio que se considere de interés para la comunidad a la que va dirigido. Para ello, se ha buscado un sistema de organización, inclusión y localización de información lo más dinámico, fácil y rápido posible, optándose por un sistema de base de datos relacionadas que permite cumplir con estos requisitos.

El portal está diseñado con la tecnología denominada Postnuke, pues así se consigue que la tecnología más reciente esté disponible a los docentes de manera clara, sencilla y útil. Además con este lenguaje, el portal se convierte en una herramienta mucho más dinámica, permitiendo que el mantenimiento por parte del webmaster sea menor y que, por el contrario, sea mayor la presencia del usuario, pues es él quien decide que noticias ver, como quiere la presentación de 
las mismas, etc. La inclusión de enlaces, contenidos educativos, reseñas, etc, es hecha directamente por el usuario. El gestor de la página se limita a validar dichos recursos. Para velar por la calidad de los contenidos que se inserten en el Portal en un futuro se creará un comité de normalización y de evaluación que asegure la idoneidad de los recursos incluidos y la determinación de los más aptos para ser incluidos y estar disponibles en la red.

\section{Estructura de los contenidos}

El portal se estructura en diversas secciones (figura 1). La sección Noticias ocupa la gran parte central, y presenta las noticias más interesantes y de última aparición. Permite al usuario estar informado cumplidamente de todo aquello que afecte al ámbito de la educación: celebración de congresos, encuentros de investigadores, trabajos personales presentados por los docentes, convocatorias educativas, iniciativas de institutos de la región en cuanto a metodología didáctica, noticias institucionales de la Consejería de Educación, Ciencia y Tecnología etc. Todas estas noticias están clasificadas por temas en un histórico de noticias, lo que permite rescatarlas en cualquier momento. La sección Enlaces, situada debajo, recoge otras noticias que por su condición no son destacadas en la zona central, pero que sí merecen especial tratamiento, normalmente porque están dirigidas a usuarios más especializados o constituyen referencia sinformativas, pero de menor valor. Así mismo, también son ordenadas por número de visitas.

Las otras dos divisiones que tiene el portal son dos columnas a ambos lados de la zona central. En la columna izquierda se ofrecen enlaces a los diversos módulos que tiene el portal, entre los cuales hemos de destacar: Noticias, Publicaciones, Calendario de Actividades, Legislación, Foros, Descargas educativas, etc. De entre estos módulos, especial importancia tiene la sección denominada Foros. Este módulo consta de un foro programado en lenguaje "php" que proporciona a la comunidad educativa un ágora donde expresar todo tipo de opiniones sobre el sistema educativo extremeño. Entre los temas que se tratan en el foro, pueden citarse la evaluación de la calidad docente, críticas al mismo, soluciones cooperativas de los docentes, etc. Otras secciones a las que se accede desde la columna izquierda del portal son:

- Enlaces educativos, donde se recogen todas las noticias, cursos, materiales $\mathrm{y}$, en general, cualquier otro recurso existente son recogidos, pero cuyo acceso se ha diseccionado en varias subsecciones para poder acceder de una manera más compresible y directa:

- Etapas educativas: educación infantil, educación primaria, educación secundaria, bachillerato, ciclos formativos.

- Necesidades educativas especiales: trastornos y deficiencias.

Scire. $9: 2$ (jul.-dic. 2003) 161-168. 


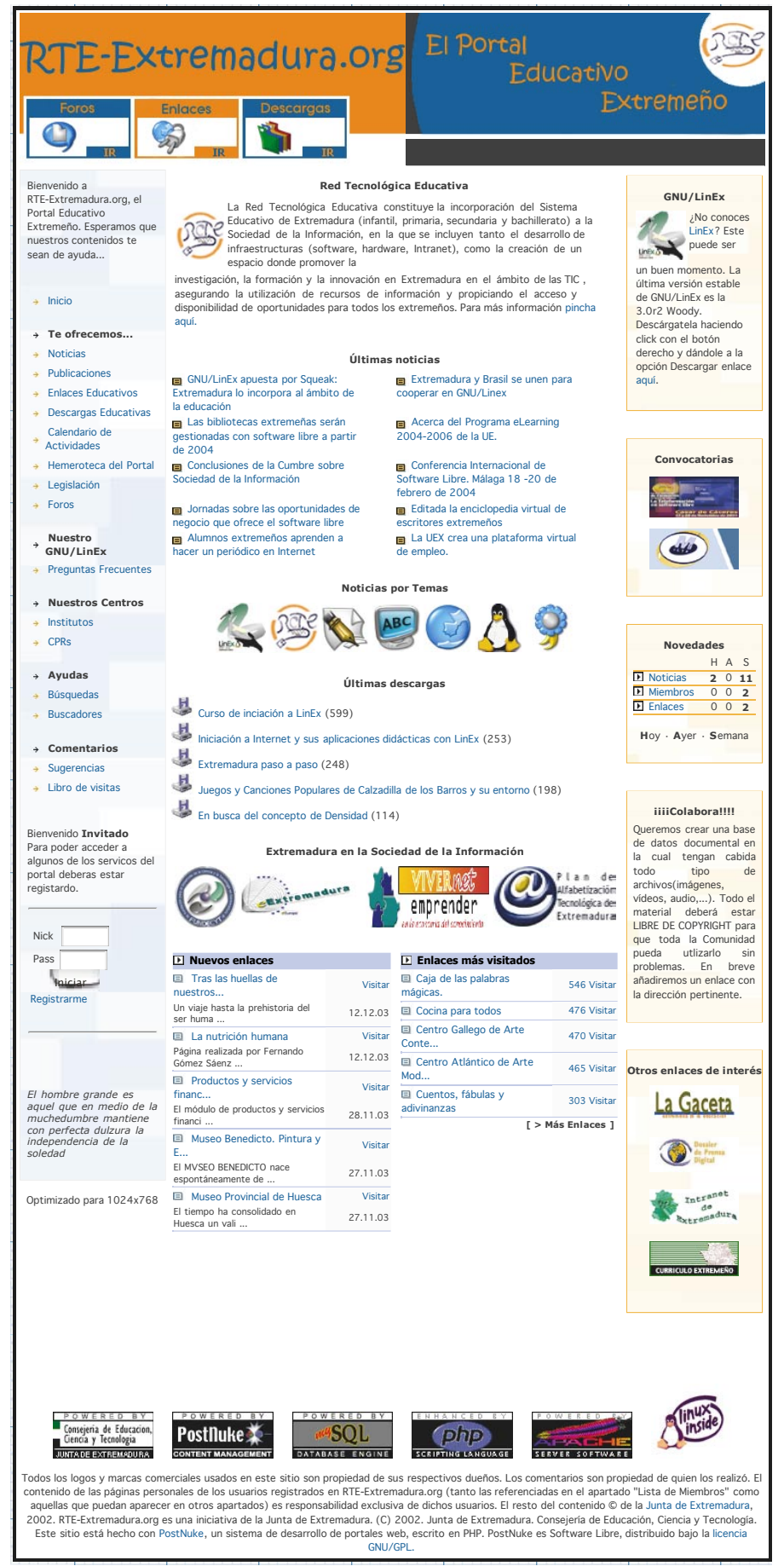

Figura 1. Página de inicio del portal 
- Escuela integradora: temas transversales y actividades extraescolares.

- Sala de lectura: publicaciones, editoriales, librerías, bibliotecas y museos.

- Formación: autoaprendizaje, software educativo y eventos.

- Orientación: seguridad en Internet, guía de peques, orientación universitaria, listas y foros, y buscadores especializados.

- Nuestros centros: directorio de la Consejería de Educación, Ciencia y Tecnología, CPRs de España, y el Mapa de CPRs de Extremadura y centros educativos de Extremadura.

- Legislación: legislación básica, convocatorias y subvenciones.

- Descargas Educativas, donde los docentes suben contenidos realizados por ellos mismos, bien como material curricular, bien como prácticas ejercidas en su docencia con la colaboración de sus alumnos. Podemos señalar como ejemplos el periódico infantil Rayuela, Extremadura Paso a Paso, etc.

- Nuestros Centros, donde se recoge el mapa educativo extremeño, con los Colegios, I. E. S., los denominados C. P. R. o Centros de Profesores y Recursos, y los Centros de Formación Profesional. En todos ellos, además de su localización geográfica, se indica su dirección postal, teléfono y correo electrónico. Además, hay que destacar que todos ellos disponen de un espacio de $100 \mathrm{Mb}$ en un servidor para alojar su propia página web, donde pueden incluir sus propios contenidos, documentación administrativa - actas, presupuestos etc. - y pública - notas, partes de asistencia, calendario de actividades, etc.

- Comentarios, dónde los usuarios, además de disponer de un libro de firmas, tienen a su disposición un formulario de sugerencias dónde expresar sus inquietudes, iniciativas, contribuciones, críticas etc.

En la columna de la derecha, existen otras secciones entre las que cabe destacar por su repercusión internacional el referido a GNU/Linex - el sistema operativo basado en Linux, más en concreto en la distribución Debian con entorno gráfico Gnome-, que está implantado en toda la Comunidad Autónoma Extremeña, que cuenta con un parque de más de 60.000 ordenadores, y con más de 300.00 usuarios activos. Entre otras cosas en este módulo podemos descargar el sistema operativo completo, acceder a manuales, a las preguntas más frecuentes (FAQ), y a foros especializados, dirigidos tanto a usuarios básicos, como a administradores de centros, docentes etc.

Como última aportación el portal genera un Boletín electrónico, denominado Educación y TIC, que se envía diariamente a más de 455 usuarios, en el se reco- 
gen las noticias más destacadas en el ámbito de la educación tanto a nivel regional y nacional como internacional.

La educación, la escasa existencia en la red de espacios que aglutinen y aúnen a las personas que trabajan en la construcción del sistema educativo extremeño, donde puedan intercambiar sus ideas, experiencias y puedan hacer sus aportaciones para el enriquecimiento colectivo, nos ha llevado a concebir este Portal que sea punto de encuentro y referencia de la Educación.

\section{Bibliografía}

Clark, Judith. Subjects portals. // Ariadne Issue. 29 (2001). URL: <http://ariadne.uc.uk/ issue29/clark/intro.html>. Consultado: 2002-10-30.

Lefébre, Philippe (2001). Les portails d'acces à l'information. // Documentaliste-Sciences de l'information. 38:3-4 (2001), 188-196.

Miller, Paul (2001). The concept of the Portal. // Ariadne Issue. 30 (2001). URL: < http: //www.ariadne.ac.uk/issue/portal/intro.html>. Consultado: 2002-10-30.

Pérez de Leza, J. (2000). El valor añadido de un portal. // Ecomm. 13 (2000). URL: < http: //www.ecommdigital.com/printasrt.ctm?ID=342>. Consultado: 2002-10-30.

Red Tecnológica Educativa. URL: http://www.rte-extremadura.org. Consultado: 200210-30. 